\title{
Iniquidades nas contribuições das fontes de financiamento da saúde na Pesquisa de Orçamentos Familiares no Brasil
}

Inequities in the contributions of health financing sources in the Family Budget Survey in Brazil

Inequidades en las contribuciones de las fuentes de financiamiento de la salud en la Encuesta de Presupuestos Familiares en Brasil

Marcelo Armijos Briones ${ }^{1}$

Fernando Pires de Sousa ${ }^{2}$

\begin{abstract}
Resumo
Objetivo: analisar a incidência no financiamento (FIA, em inglês), tanto estrutural como efetiva, das fontes de financiamento da saúde, encontradas na Pesquisa de Orçamentos Familiares (POF) de 2008/2009 e a distribuição dessas contribuições na população segundo seu poder de consumo. Metodologia: foram utilizados os dados da Pesquisa de Orçamentos Familiares de 2008/2009 como fonte de dados, de onde foram obtidas as fontes de financiamento (públicas e privadas) do sistema de saúde no Brasil. Essas fontes foram analisadas em referência ao poder de consumo per capita da população para avaliar a progressividade, regressividade ou proporcionalidade delas, mediante o uso de índices de concentração, Gini e Kakwani. Para isso, o estudo usou o pacote estatístico Stata 12 e o programa estatístico $A D e P T$ do Banco Mundial. Resultados: os pagamentos diretos em saúde mantiveram uma distribuição regressiva, enquanto os pagamentos com planos de saúde e impostos diretos foram progressivos e proporcionais, respetivamente. A consolidação das três fontes de financiamento foi avaliada como proporcional, por ter um índice de Kakwani de -0,0349. O índice de Gini demonstrou que os gastos feitos com saúde são ainda menos equitativos $(0,609)$ que a distribuição geral dos recursos $(0,598)$ dentre a população. Conclusões: o orçamento público não é suficiente para suprir todas as necessidades do SUS e as contribuições das fontes de financiamento não são progressivas, e essa é uma das possíveis causas do aumento da pobreza e das catástrofes financeiras da população brasileira.
\end{abstract}

Palavras-chave: Brasil. Pobreza. Financiamento da saúde. Economia da saúde. Equidade.

\begin{abstract}
Objective: to analyze the Incidence in Financing (FIA), both structural and effective, from the sources of health financing, found in the Family Budget Survey 2008/2009 and the distribution of these contributions in the population according to their power of consumption. Methodology: The data from the Family Budget Survey 2008/2009 were used as a data source, from where the sources of financing (public and private) of the health system in Brazil were obtained. These sources were analyzed in reference to the per capita consumption power of the population to assess their progressivity, regressivity or proportionality, by using

\footnotetext{
1 Doutor em Saúde Pública; professor titular, Universidad Regional Autónoma de los Andes, Carrera de Odontología, Ambato, Tungurahua,Ecuador. https://orcid.org/0000-0002-5500-4768. E-mail: ua.fernandoarmijos@uniandes.edu.ec

2 Doutor em Economia; professor titular, Universidade Federal do Ceará, Departamento de Teoria Econômica, Fortaleza, Ceará, Brasil. https://orcid.org/0000-0001-8334-5407.E-mail: fjpires.s@ufc.br
} 
indices of concentration, Gini and Kakwani. For this, the study used the Stata 12 and $A D e P T$ statistical programs of the World Bank. Results: direct payments in health maintained a regressive distribution, while payments with health insurance and direct taxes were progressive and proportional respectively. The consolidation of the three financing sources was determined as proportional by having a Kakwani index of -0.0349 . The Gini index showed that health expenditures are even less equitable than the general distribution of resources among the population (0.598). Conclusions: the public budget is not sufficient to meet all the needs of the Unified Health System and the contributions of the financing sources are not progressive and this may be one of the causes of the increase in poverty and financial catastrophes of the Brazilian population.

Keywords: Brazil. Poverty. Financing of health care. Health economics. Equity.

\section{Resumen}

Objetivo: analizar la Incidencia en el Financiamiento (FIA, en inglés), tanto estructural como efectivo, de las fuentes de financiamiento de la salud, encontradas en la Encuesta de Presupuestos Familiares de 2008/2009 y la distribución de esas contribuciones en la población según su poder de consumo. Metodología: fueron usados los datos de la Encuesta de Presupuestos Familiares de 2008/2009 como fuente de datos, donde se obtuvo las fuentes de financiamiento (públicas y privadas) del sistema de salud en Brasil. Esas fuentes fueron analizadas en referencia al poder de consumo per cápita de la población para evaluar la progresividad, regresividad o proporcionalidad de estas, mediante el uso de índices de concentración, Gini y Kakwani. Para esto, el estudio utilizó los programas estadísticos Stata 12 y ADePT del Banco Mundial. Resultados: los pagos directos en salud mantuvieron una distribución regresiva, mientras que los pagos con seguros de salud e impuestos directos resultaron ser progresivos y proporcionales respectivamente. La consolidación de las tres fuentes de financiamiento fue determinada como proporcional por tener un índice de Kakwani de -0.0349. El índice de Gini demostró que los gastos en salud son aún menos equitativos que la distribución general de los recursos entre la población (0.598). Conclusiones: el presupuesto público no es suficiente para suplir todas las necesidades del Sistema Único de Salud y las contribuciones de las fuentes de financiamiento no son progresivas y esto puede ser una de las causas del aumento de la pobreza y de catástrofes financieras de la población brasileña.

Palabras clave: Brasil. Pobreza. Financiamiento de la salud. Economía de la salud. Equidad.

\section{Introdução}

O empobrecimento das famílias resultante dos gastos com saúde é, em grande medida, devido à falta de proteção financeira dos sistemas de saúde, definido como gastos extraordinários. Segundo a Organização Mundial da Saúde (OMS), mais de 150 milhões de pessoas se veem nessa situação ao redor do mundo (1). Porém, não apenas os gastos diretos com saúde são os responsáveis do empobrecimento familiar, condições socioeconômicas de iniquidade, especialmente no financiamento dos sistemas de saúde ao redor do mundo, são também responsáveis por esse fenômeno (2-4), que é mais 
intensificado em regiões como a América Latina, considerada uma das mais desiguais do mundo (3). Uma amostra dessa iniquidade é o financiamento dos sistemas de saúde de alguns países da região, onde o gasto público em saúde é menor do que o gasto privado (4).

O Brasil encontra-se nesse grupo de países, onde o gasto privado representou 56,7\% do gasto total em saúde (GTS), em 2015 (5). O recomendado pela OMS para os países membros da Organização Pan-americana da Saúde (OPAS) é atingir $80 \%$ de gasto público em saúde ou até 6\% do PIB para garantir a cobertura de proteção financeira (6), componente básico da cobertura universal de saúde, definida como "o acesso aos serviços de saúde de qualidade, sejam esses de promoção, prevenção, tratamento, reabilitação e cuidados sem que eles demandem dificuldades financeiras" (1). No Brasil, como em outros países da região como Equador, Bolívia, Venezuela, Colômbia, o direto à saúde está garantido constitucionalmente (7), denominando os seus sistemas de saúde públicos como universais na busca por garantir o acesso aos serviços de saúde. Porém, ainda existe uma grande desigualdade nesse acesso, o que aprofunda a iniquidade em saúde e a inequidade geral nesses países $(8,9)$.

A garantia do direito universal à saúde não oferece, pelo menos no Brasil, cobertura de proteção financeira aos seus usuários. Em 2016, segundo Vera Lucia Luiza et al (10), um em cada 17 domicílios no Brasil sofria de catástrofe financeira relacionada ao gasto feito com pagamentos diretos a serviços de saúde e compras de planos de saúde, ambas fontes privadas de financiamento da saúde (11-15). Porém, poucos estudos descrevem a magnitude da iniquidade desse financiamento do sistema de saúde brasileiro e, até 2016, existia apenas um trabalho sobre equidade no financiamento na América Latina, especificamente no Brasil (5).

Com a finalidade de contribuir com essa discussão, realizamos uma análise da equidade no financiamento, usando a análise de incidência de financiamento (FIA, sigla em inglês) (16). Esse tipo de análise procura avaliar a distribuição das contribuições ou pagamentos feitos pela população nos mecanismos ou fontes de financiamento do sistema de saúde de um país. Essa distribuição pode ser i) progressiva, situação onde a parcela da população com mais recursos contribui mais do que as que menos têm; ii) regressiva, situação onde a população mais pobre contribui mais do que a mais rica; e iii) proporcional, quando toda a população contribui na mesma proporção. Esse tipo de análise possui duas formas: estrutural e efetiva. Na primeira, é possível analisar a distribuição das contribuições 
da população para uma determinada fonte de financiamento do sistema de saúde. Na segunda forma, é possível analisar a intensidade da distribuição das contribuições para os mecanismos de financiamento mediante o uso de índices como Gini, Kakwani e de concentração, além da avaliação das curvas de concentração em relação à distribuição dos recursos disponíveis pela população $(16,17)$.

Existem várias fontes ou mecanismos de financiamento que podem ser analisados pela FIA, tanto públicas (impostos diretos e indiretos) e privadas (planos de saúde, compra direta de serviços e contribuições para seguros de saúde públicos). Nesse estudo, analisamos apenas as fontes ou mecanismos de financiamento da saúde que foi possível encontrar na base de dados da Pesquisa de Orçamentos Familiares de 2008/2009 (POF 2008/2009) (18). Dentre elas, as contribuições ou pagamentos feitos com planos de saúde, compra direta de serviços médicos e impostos diretos - à proporção que é destinada para 0 financiamento da saúde pública no Brasil, o SUS. Essa pesquisa, apesar de ter uma periocidade de 5 anos, não foi feita em 2013 pelo IBGE sem qualquer justificativa aparente, e, com isso, a base de dados utilizada é a mais atual na data da pesquisa.

O objetivo desse estudo é realizar uma análise da incidência no financiamento (FIA), tanto estrutural como efetiva, das fontes de financiamento da saúde encontradas na POF 2008/2009, para avaliar a distribuição das contribuições ou pagamentos da população segundo seu poder de consumo.

\section{Metodologia}

Nesse estudo transversal, usamos como fontes de dados a Pesquisa de Orçamentos Familiares de 2008/2009 (POF), realizada pelo IBGE (18), a qual teve como objetivo conhecer a composição orçamentária e hábitos de consumo da população por meio de uma amostra representativa do país. Essa pesquisa usou amostragem aleatória simples em dois estágios. No primeiro, foram selecionadas as unidades primárias de amostragem ou setores censitários, obtidos a partir do censo demográfico do ano 2000. No segundo, foram selecionadas as unidades secundárias de amostragem que corresponderam aos domicílios. Do total de setores censitários, foram aumentados $30 \%$ com a finalidade de corrigir domicílios que estiveram desocupados ou que recusassem a serem entrevistados. Ao final, a amostra compreendeu um total de 4.696 setores censitários e 59.548 domicílios selecionados. Os dados da POF foram disponibilizados no site oficial do IBGE em forma de 
microdados, os quais foram descarregados em formato de texto e, com assistência do complemento de DataZoom para Stata 12 (pacote para Stata para a leitura de microdados das pesquisas de base domiciliar do IBGE), foram convertidos para uma base de dados padronizada, com variáveis já deflacionadas e anualizadas. Para a realização das análises, foram usados os pesos amostrais, ou fatores de expansão da amostra, fornecidos também na base de dados. A mesma base de dados foi utilizada no programa estatístico ADePT fornecido de forma gratuita pelo Banco Mundial para realizar, entre outros cálculos, as FIAs para a criação das curvas de concentração.

As FIAs aqui apresentadas seguem a metodologia fornecida por O'Conell e Ataguba. Segundo esses autores, a FIA é uma análise destinada a avaliar a equidade no financiamento de um sistema de saúde e/ou suas fontes, que podem ser públicas ou privadas, em referência a uma medida reflexo do estilo de vida da população, ou seja, uma forma quantificável para medir o estilo de vida da população, que pode ser pelo grau de renda ou grau de consumo familiar. $(16,17)$. Para isso, é preciso identificar dentro da base de dados as variáveis que contêm essas informações.

\section{Fontes de financiamento}

As fontes públicas compreendem tributos como os impostos diretos, para cumprir obrigações fiscais que incidem sobre renda e patrimônio, e os indiretos, que incidem sobre o consumo, ou seja, são inclusos nos preços da compra de bens ou serviços. De ambos, considera-se apenas a parcela arrecadada que é destinada para a saúde. Para isso, precisase saber previamente, mediante informações oficiais ou empíricas, quanto do arrecadado em impostos é destinado para a saúde. Já as fontes privadas são despesas diretas com saúde feitas pelas pessoas, como a compra de planos de saúde privados. Nesse estudo, foram consideradas apenas as fontes encontradas na POF 2008/2009.

Também só foi possível analisar os impostos diretos pagos pela população, dos quais, segundo Ugá e Santos (19), 10,6\% do arrecadado é destinado ao financiamento do Sistema Único de Saúde (SUS) no Brasil. Esses impostos se encontravam contidos numa variável denominada impostos, dentro da base de dados. Os impostos que constavam nessa variável foram o Imposto sobre a Propriedade Predial e Territorial Urbana (IPTU); Imposto sobre a Propriedade Territorial Rural (IPTR); Imposto sobre Propriedade de Veículos Automotores (IPVA); Impostos Sobre Serviços (ISS); Imposto de Renda (exercício anterior); Imposto de 
Renda de trabalhadores (públicos e privados), empregadores, empresários; e outras deduções. Dessa variável, foi obtida a percentagem nomeada e criada a variável que continha a fonte de financiamento impostos diretos.

Para as fontes de financiamento privadas, no caso dos pagamentos diretos com saúde, foram somados todos os itens com gastos em saúde, com excepção dos pagamentos feitos com planos de saúde, nos quais as pessoas entrevistadas declararam ter pagado alguma quantia. O resultado foi a variável que continha a fonte de financiamento de pagamentos diretos, denominada como out of pocket. No caso das compras de planos de saúde, a base de dados mantinha uma variável única para essa fonte de financiamento de forma desagregada; com essa informação, foi criada a variável que continha a fonte de financiamento de planos de saúde privados denominada como plano saúde.

Todos os gastos feitos com saúde, encontravam-se deflacionados na base de dados e já disponíveis de forma anual, como exige a metodologia para FIA.

Para a FIA é preciso também, uma medida reflexo do estilo de vida da população, como a renda ou consumo, para avaliar qual proporção é destinada para cada fonte de financiamento e sobre quem recai o maior peso desse financiamento. Para este estudo, tomou-se o consumo domiciliar per capita como medida do estilo de vida da população e denominada de capacidade de pagamento (CDP). Ao usar o consumo, foi preciso também considerar as economias de escala, com o princípio de que nem todas as pessoas consumem na mesma quantia dentro do domicílio. Para isso foi usada a escala de equivalência da OMS, usada na metodologia por eles criada para medir a cobertura de proteção financeira no progresso até a cobertura universal de saúde. A escala de 0,56 foi obtida com base em um estudo feito em 59 países, incluso o Brasil (20). Dessa forma, o tamanho do domicílio ou a quantidade de moradores foi elevada para a escala de equivalência, depois o resultado foi dividido para o consumo total do domicílio (a soma de todos os consumos familiares feitos durante o ano que durou a pesquisa) e assim se obteve a capacidade de pagamento per capita ou CDP.

A metodologia empregada é flexível quanto à divisão da população por quartis, a qual pode ser por quintil ou decil. Neste estudo, dividimos a população por quintil da CDP, desde o $20 \%$ mais pobre até o $20 \%$ mais rico da população. 


\section{FIAs estruturais e efetivas}

Já com as variáveis das fontes de financiamento e da CDP, foi possível realizar as FIAs nas suas duas formas. Para ambas, foi usado o complemento fia para Stata 12, fornecido por Ataguba (16), com as seguintes sintaxes:

1. FIA estrutural: fia out_of_pocket plano_saude impostos_diretos type (quantile) hweight (FEXP) rank (CDP) nq (5)

Onde fia é o nome do comando do complemento específico, seguido das fontes de financiamento encontradas na base de dados; type é o tipo de análise (quantile) para FIA estrutural; rank é a variável pela qual é dividida a população, no caso, por CDP; nq é a eleição da divisão da população, no caso, por quintil da CDP (5).

O resultado dessa análise é a proporção e distribuição dos gastos feito pela população em cada fonte de financiamento segundo a CDP.

2. FIA efetiva: fia out_of_pocket plano_saude impostos_diretos type (index) hweight (FEXP) rank (CDP) nq (5)

Onde fia é o nome do comando do complemento específico seguido das fontes de financiamento encontradas na base de dados; type é o tipo de análise (index) para FIA específica; rank é a variável pela qual é dividida a população, no caso, por CDP; nq é a eleição da divisão da população, no caso, por quintil da CDP (5).

O resultado dessa análise é uma tabela com os índices de concentração, Kakwani e de Gini, que avaliam a equidade no financiamento dos sistemas de saúde. Os quais se definem da seguinte forma:

1. O índice de concentração pode variar de -1 (situação onde os domicílios mais pobres arcam com toda a fonte de financiamento da saúde) até +1 (situação inversa, onde os lares mais ricos são os que financiam). Quando negativo, indica que a curva de concentração se encontra acima da linha de igualdade perfeita (linha de 45 graus) e, quando positivo, significa que a curva de concentração para a fonte de financiamento está abaixo da linha de equidade perfeita.

2. O índice de Kakwani é uma medida de resumo sobre a progressividade e pode ir desde -2 , relatando uma fonte regressiva total $\mathrm{e}+1$, uma fonte progressiva, e é proporcional quando o índice é zero (21).

3. O coeficiente de Gini pode ir desde 1, que expressa uma desigualdade perfeita, e zero, uma igualdade perfeita. 
A medida resumo de Kakwani avalia a distribuição do financiamento de forma numérica e fornece uma leitura precisa dessa distribuição. Porém, o desenho de curvas de concentração contribui melhor para analisar de forma gráfica a dominância das curvas ao respeito da curva de Lorenz - curva se refere à distribuição da riqueza da população estudada ou à medida reflexo do estilo de vida CDP - de distribuição dos recursos ou curva da CDP. Para desenhar essas curvas, fizemos uso do programa ADePT do Banco Mundial, no qual, inserindo as variáveis que continham as fontes de financiamento, CDP, fator de expansão e o quartil desejado para dividir a população, forneceu as curvas de concentração de cada fonte de financiamento em referência à CDP e à linha de igualdade perfeita, ou de 45 graus. As curvas são interpretadas pela sua localização em relação à curva da CDP e à linha de 45 graus. Quando a curva de concentração de alguma fonte de financiamento está acima da curva da CDP, essa fonte é considerada como progressiva; quando se encontra baixo da curva da CDP é considerada como regressiva; e quando se encontra muito próxima ou juntas, é considerada como proporcional.

\section{Considerações éticas}

O estudo foi realizado com bases de dados secundárias e públicas, no qual é impossível a identificação de qualquer participante na pesquisa e, portanto, não foi necessário consentimento livre e esclarecido.

\section{Resultados}

Distribuição dos gastos com saúde feitos pela população segundo a POF 2008/2009.

Os gastos com saúde na POF 2008/2009 representam uma grande parcela de recursos para o financiamento privado do sistema de saúde no Brasil e são os que têm o maior peso no orçamento familiar. Na tabela 1, é possível observar como estão distribuídas essas despesas, segundo as variáveis que constam na POF. As despesas com medicamentos são as que demandam maiores volumes de recursos, pois concentram $48,63 \%$ do total de gastos privados com saúde ( $\mathrm{R} \$ 106$ bilhões), acompanhado pelos realizados com planos de saúde privados (29,78\%). Quando distribuímos esses gastos por quintil da CDP, verificamos o peso que cada um exerce sobre a capacidade de consumo domiciliar, com destaque para os medicamentos nos domicílios mais pobres, especialmente 
nos quintis 1 e 2, comprometendo 9,64\% e 8,73\%, respetivamente, enquanto os domicílios mais ricos gastaram apenas 3,96\% da sua CDP, fazendo dessa uma distribuição regressiva ao respeito desse item específico. O contrário, e esperado, foi achado na distribuição dos pagamentos feitos com planos de saúde. Nesse item, é possível observar uma distribuição progressiva dos pagamentos feitos em compra de planos de saúde. O quintil mais rico da população é o que destina maior parte da sua CDP, 4,42\%, valor superior em 16 vezes ao dos domicílios mais pobres $(0,27 \%)$. A percentagem restante é o resultado da agrupação dos demais itens levantados na POF 2008/2009 (que podem ser disponibilizados em forma desagregada mediante petitório).

Essa distribuição é específica para todos os itens levantados durante a pesquisa. Já a respeito das fontes de financiamento do sistema de saúde, que foram achadas na POF 2008/2009, duas privadas - pagamentos diretos e planos de saúde - e uma pública pagamentos de impostos diretos que financiam a saúde - se encontram detalhadas por tipo de FIA.

\section{FIA estrutural}

A primeira fonte analisada são os pagamentos diretos em relação aos quintis da CDP da população (gráfico 1). Para essa modalidade de financiamento, o gráfico representa uma escala meramente regressiva, na qual os domicílios com menor CDP destinam mais recursos para financiar essa fonte pública do que os domicílios mais ricos do país. Ainda mais preocupante é a magnitude do gasto dos dois quintis mais pobres (1 e 2), em ambos supera $10 \%$ do total da CDP. 
Gráfico 1. Distribuição por quintis dos pagamentos com saúde como proporção da CDP das fontes de financiamento que constam na POF 2008/2009 - FIA estrutural

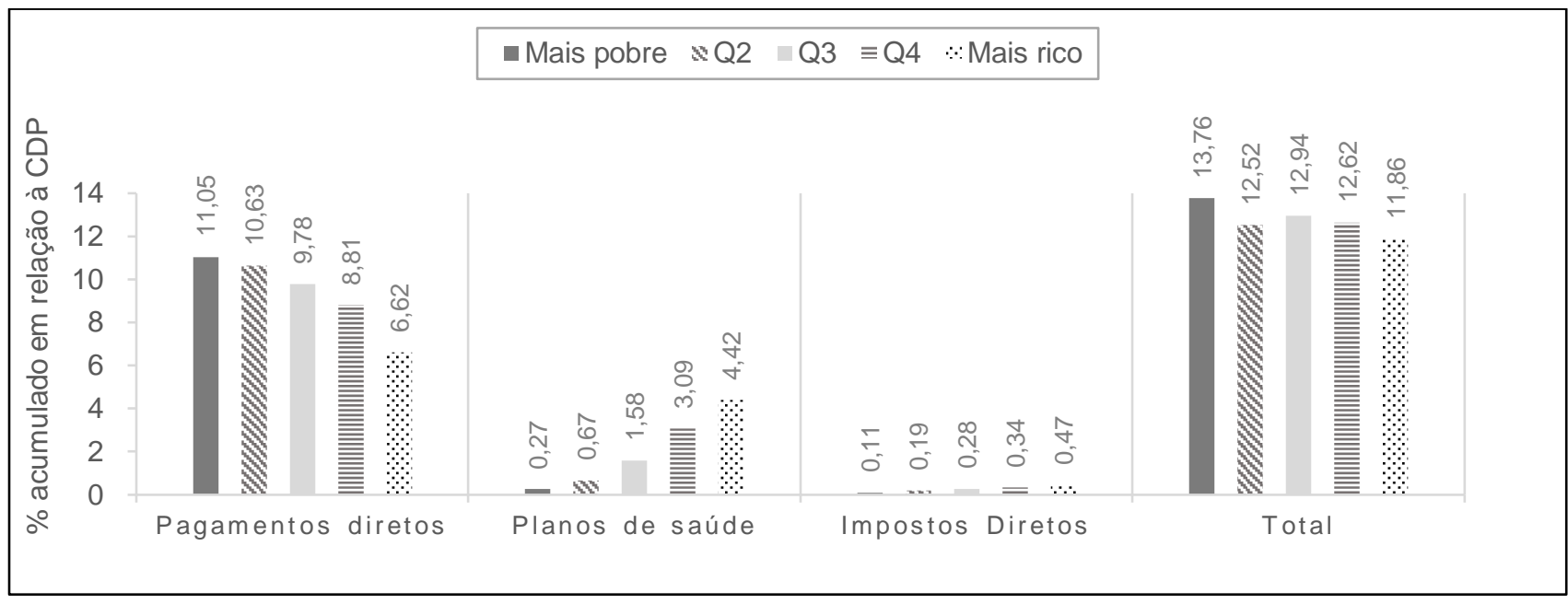

Fonte: elaboração própria.

${ }^{*} \mathrm{CDP}$ : capacidade de pagamento per capita.

A segunda fonte se refere aos pagamentos feitos com planos de saúde (gráfico 1). Como era esperado, e mantendo uma relação com a distribuição (tabela 1), os domicílios com menor renda destinaram menor quantidade de recursos para adquirir essa forma de atendimento do que os domicílios mais ricos: $0,27 \%$ da CDP do quintil mais pobres e 4,42\% no mais rico, evidenciando uma clara progressividade desse mecanismo de financiamento. Porém, se evidencia também um esforço da classe média, representada nos quintis 3 e 4 , para adquirir planos de saúde, destinando entre 1,5\% e mais de $3 \%$ dos seus recursos em planos de saúde.

Tabela 1. Distribuição dos pagamentos diretos em saúde no Brasil que constam na POF 2008/2009

\begin{tabular}{l|c|c|c|c|c|c|c}
\hline \multirow{2}{*}{$\begin{array}{l}\text { Tipos de } \\
\text { pagamentos }\end{array}$} & \multicolumn{3}{|c|}{$\begin{array}{c}\text { Gasto total em } \\
\text { bilhões de reais }\end{array}$} & \multicolumn{5}{|c}{ Distribuídos por quintil da CDP* } \\
\cline { 2 - 8 } & $\mathbf{N}$ & $\%$ & Mais pobre & $\mathbf{Q 2}$ & $\mathbf{Q 3}$ & $\mathbf{Q 4}$ & Mais rico \\
\hline Remédios & 51,9 & 48,6 & 9,6 & 8,7 & 7,7 & 6,4 & 3,9 \\
\hline Planos de saúde & 31,8 & 29,8 & 0,3 & 0,7 & 1,6 & 3,1 & 4,4 \\
\hline Outros gastos** & 22,4 & 21,6 & 1,4 & 1,9 & 2,1 & 2,4 & 2,7 \\
\hline Total & $\mathbf{1 0 6 , 1}$ & $\mathbf{1 0 0}$ & \multicolumn{7}{|c|}{} \\
\hline
\end{tabular}

Fonte: elaboração própria.

*CDP: capacidade de pagamento per capita.

** Gastos com hospitalização, dentista, próteses, materiais e outros. 
Quanto à terceira fonte de financiamento encontrada - a proporção de impostos diretos pagos pela população que é destinado para o financiamento da saúde -, não representou um grande gasto, apenas $0,10 \%$ no quintil mais pobre e $0,46 \%$ no mais rico, caracterizando-se assim, como uma fonte progressiva (gráfico 1).

A consolidação das três fontes de financiamento resultou com uma tendência teoricamente regressiva, embora não tão marcada como a distribuição dos pagamentos diretos. Apesar do quintil 2 não seguir o padrão, é observado que o quintil mais rico $(11,86 \%)$ contribui menos que o quintil mais pobre $(13,76 \%)$.

\section{FIA efetiva}

O gráfico 2 apresenta as curvas de concentração para as fontes de financiamento privadas e públicas do sistema de saúde no Brasil, encontradas na POF 2008/2009, as quais corroboram o observado na FIA estrutural: a regressividade dos pagamentos diretos com saúde e a progressividade dos planos de saúde e impostos diretos. No primeiro caso, a curva de concentração está localizada acima da curva de Lorenz da distribuição dos recursos dentre a população ou de CDP, o que mostra a sua regressividade. No segundo caso, pode ser observado o contrário, as curvas de concentração das contribuições, ou pagamentos com planos de saúde e impostos indiretos, encontram-se abaixo da curva da CDP. As curvas de concentração também mostram a distância que existe entre alguma fonte de financiamento ou até mesmo a CDP e a igualdade perfeita ou ideal, fato que possibilita uma ideia mais abrangente da inequidade das fontes de financiamento da saúde e da distribuição geral dos recursos de um país. 
Gráfico 2. Curvas de concentração das fontes de financiamento da saúde que constam na POF 2008/2009 - FIA efetiva

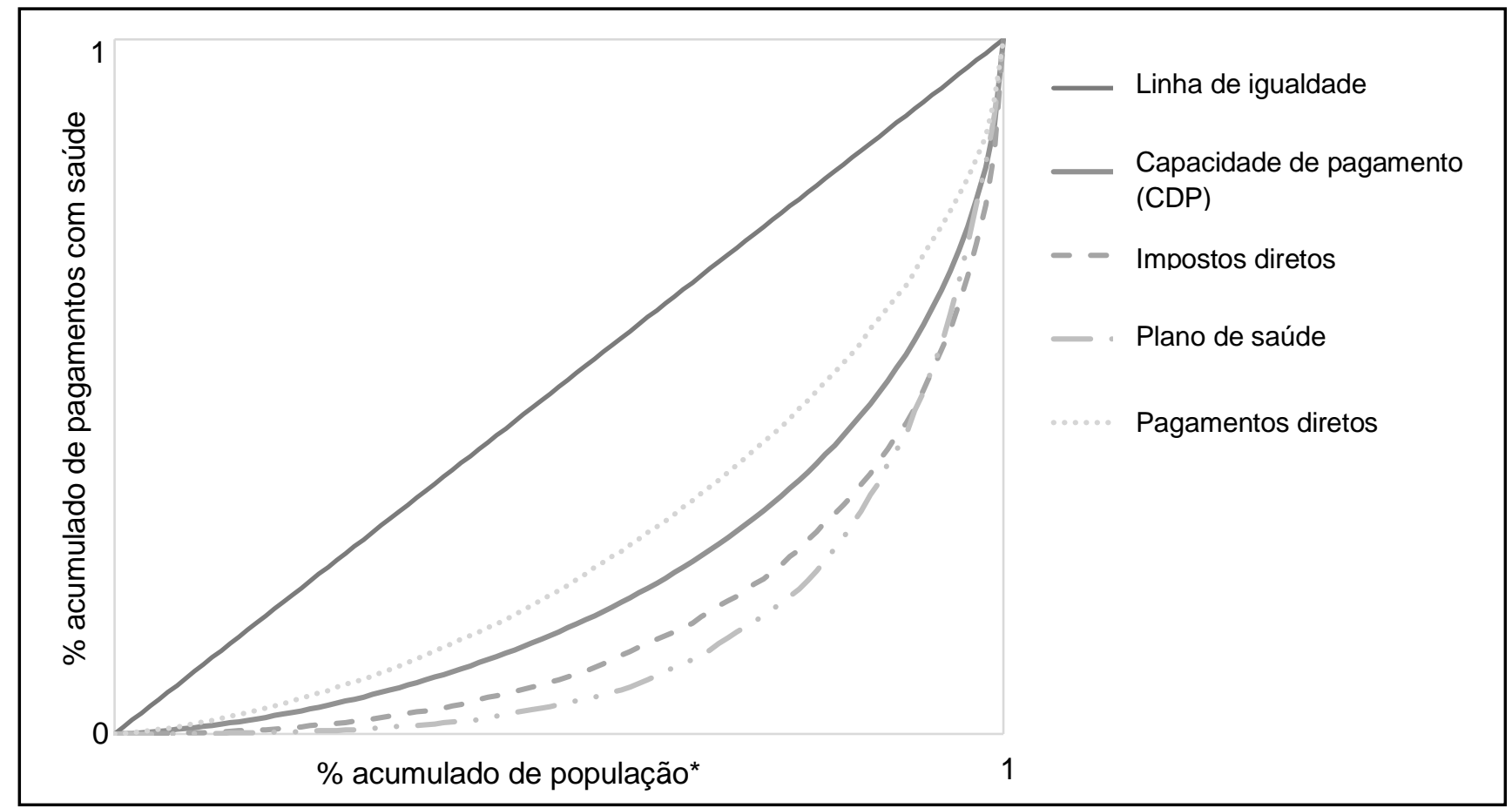

Fonte: elaboração própria.

*Agregado do mais pobre até o mais rico.

A representação numérica das curvas de concentração, assim como a medida resumo dos índices de Kakwani e de Gini, são mostrados na tabela 2, onde se pode observar uma reafirmação dos resultados encontrados nas análises anteriores. O índice de Kakwani é negativo para os pagamentos diretos $(-0,11)$, avaliando essa fonte como regressiva, e as outras duas fontes - planos de saúde e impostos - com valores positivos $(0,09$ e 0,13, respetivamente), avaliando-as como progressivas. Já a consolidação das três fontes segundo Kakwani, teoricamente poderia ser considerada como uma consolidação regressiva pelo índice ser negativo $(-0,034)$, porém, a sua proximidade do zero fez com que seja considerada como proporcional. Ou seja, a consolidação das três fontes de financiamento do sistema de saúde no Brasil, encontradas na POF 2008/2009, é proporcional: toda a população paga uma proporção similar da sua CDP para o financiamento da saúde.

O índice de Gini (tabela 2), que já era alto para a distribuição da $\operatorname{CDP}(0,598)$, ficou ainda maior na distribuição das contribuições de todas as fontes de financiamento da saúde $(0,609)$, mostrando de forma numérica o grau de inequidade no financiamento da saúde no Brasil. 
Tabela 2. Índices de concentração, Gini e Kakwani das fontes de financiamento do sistema de saúde brasileiro que constam na POF 2008/2009 - FIA efetiva

\begin{tabular}{|c|c|c|c|c|c|c|}
\hline $\begin{array}{l}\text { Quintis da } \\
\text { CDP* }^{\star}\end{array}$ & $\begin{array}{l}\text { Distribuição } \\
\text { da CDP na } \\
\text { população }\end{array}$ & $\begin{array}{l}\text { Impostos } \\
\text { diretos }\end{array}$ & $\begin{array}{l}\text { Plano de } \\
\text { saúde }\end{array}$ & $\begin{array}{l}\text { Pagamentos } \\
\text { diretos }\end{array}$ & $\begin{array}{l}\text { Total de } \\
\text { pagamentos }\end{array}$ & $\begin{array}{l}\text { Distribuição } \\
\text { das despesas } \\
\text { com saúde }\end{array}$ \\
\hline Mais pobre & 2,4 & 0,7 & 0,3 & 3,9 & 2,7 & 2,3 \\
\hline 2 & 5,6 & 3,2 & 1,6 & 8,0 & 6,0 & 5,5 \\
\hline 3 & 10,0 & 7,4 & 5,6 & 13,7 & 11,2 & 9,9 \\
\hline 4 & 18,3 & 16,8 & 16,2 & 21,5 & 19,8 & 18,1 \\
\hline Mais rico & 63,7 & 71,8 & 76,4 & 52,8 & 60,2 & 64,2 \\
\hline Total & 100,0 & 100,0 & 100,0 & 100,0 & 100,0 & 100,0 \\
\hline $\begin{array}{l}\text { Coeficiente de } \\
\text { Gini }\end{array}$ & 0,5983 & - & - & - & - & 0,6092 \\
\hline $\begin{array}{l}\text { Índice de } \\
\text { concentração }\end{array}$ & - & 0,6913 & 0,7292 & 0,4867 & 0,5634 & - \\
\hline $\begin{array}{l}\text { Índice de } \\
\text { Kakwani }\end{array}$ & - & 0,0930 & 0,1310 & $-0,1115$ & $-0,0349$ & - \\
\hline
\end{tabular}

Fonte: elaboração própria no programa ADePT, com dados da POF 2008/2009.

${ }^{*} \mathrm{CDP}$ : capacidade de pagamento per capita.

\section{Discussão}

Os dados desse estudo mostraram que o item onde a população teve o maior gasto em saúde é com medicamentos. A distribuição entre quintis da CDP foi claramente regressiva, e se manteve essa distribuição quando somados os demais itens dentro da fonte de financiamento da saúde correspondente aos pagamentos diretos ou out of pocket. Dados similares foram achados em estudos que mesuraram a pobreza e as catástrofes financeiras devido aos gastos com saúde (12-14, 22-24).

Boing et. al., com dados da mesma POF, evidenciaram que houve um aumento de até $2,3 \%$ de pobreza na população brasileira (12) e que até 18,2\% sofreu de catástrofes financeiras devido aos gastos com saúde (22). Em ambos estudos, o principal gasto com saúde também foi com medicamentos. É preciso deixar claro que os recursos destinados para a compra de medicamentos por parte da população é um dos responsáveis pelo empobrecimento familiar devido a gastos com saúde, porém, não é o único: os gastos com planos de saúde potencializam esse fenômeno. Os dados de nosso estudo mostram que planos de saúde é segundo maior gasto e, que, embora com uma distribuição progressiva, quando se junta com o gasto feito com medicamentos, supera os $10 \%$ do gasto total em saúde em relação ao consumo total das famílias. Essa percentagem é um dos limites usados 
por Boing et. al. para determinar as catástrofes financeiras na população devido aos gastos com saúde (22).

É evidente que a compra de planos de saúde não representa uma garantia de proteção financeira na população usuária desse serviço, ao contrário, resulta ser um precursor de empobrecimento entre os quintis 2 e 3, que estão mais próximos das linhas de pobreza. Uma das possíveis causas dos planos de saúde estarem aumentando a pobreza da população poderia ser a isenção por lei que as empresas têm para o fornecimento de medicamentos para os seus usuários (Lei 9.656/1998), os quais, além de pagar pelo serviço, devem comprar seus medicamentos receitados. Além disso, essa parcela da população também paga os impostos que financiam o SUS, resultando em um gasto duplo para os usuários e em grande benefício para as empresas fornecedoras desses serviços, em detrimento do sistema público. Com isso, fecha o círculo que possivelmente faz as pessoas contratarem planos de saúde privados, pois uma das consequências da falta de orçamento para a saúde pública é a diminuição na oferta de serviços (25).

Dizemos que esse processo resulta no detrimento do serviço público porque este é financiado pela arrecadação de impostos e parece que no Brasil existe um certo estímulo governamental para que as pessoas contratem planos de saúde, como, por exemplo, a isenção de impostos na contratação de planos de saúde, cujas renúncias ficais podem chegar a até $R \$ 240$ bilhões, ou visto de outra forma, até 30,6\% do orçamento anual do Ministério da Saúde brasileiro (26). Também há casos em que servidores públicos, quando demonstram a contratação de um plano de saúde, recebem uma compensação monetária ou auxílio de saúde por parte das instituições públicas onde laboram. Esse cenário parece ser uma aceitação governamental de que o serviço que eles são obrigados a fornecer não dá conta de toda a população que o financia $(25,27)$. Outra distorção é o SUS cobrir as falências do sistema complementar. Por exemplo, um estudo feito no estado de Minas Gerais relata que $31 \%$ das solicitações de medicamentos excepcionais eram de usuários do sistema complementar, e os gastos do governo do estado para cobrir esses pedidos representaram $37 \%$ do gasto total com esse tipo de medicamento, e que, em média, esses usuários eram mais custosos do que os usuários sem planos de saúde (28).

Não são apenas as renúncias fiscais com planos de saúde que enfraquecem o financiamento do sistema de saúde público no Brasil e que resulta em gastos regressivos em saúde, como demonstra este estudo. Existem políticas públicas fiscais, como a Emenda 
Constitucional EC/95, aprovada em 2016 (29), que congela os recursos do Governo federal destinados à saúde por 20 anos no patamar do ano da aprovação, o que sem dúvida enfraquecerá ainda mais o financiamento público da saúde. Com essa medida, espera-se que o orçamento do Governo federal para o gasto público em saúde passe de 1,46\% do PIB em 2017, para 0,84\% em 2036 (30), enfraquecendo ainda mais o orçamento já insuficiente para a saúde pública no Brasil e forçando o gasto privado em saúde a continuar sendo o maior financista do sistema de saúde no país.

Os dados aqui apresentados, ao serem relacionados com estudos que mensuraram a proteção financeira no Brasil com a mesma base de dados (POF 2008/2009), são uma evidência de que a iniquidade nas contribuições das fontes de financiamento da saúde está intimamente relacionada com o aumento da pobreza devido aos gastos com saúde, proporcionando maior sustento científico na determinação das causas desse fenômeno, que atinge mais de $10 \%$ da população brasileira $(13,22)$. Os dados deste estudo, apesar de ter como limitante a falta da análise da parcela de impostos indiretos arrecadados que é destinada para o financiamento do SUS, contribui para, com as fontes aqui analisadas, comparar no tempo a distribuição das contribuições para os diversos mecanismos de financiamento da saúde no Brasil. A saber, no estudo de Ugá e Santos (19), com dados da POF 2002/2003, os gastos da população com pagamentos diretos em saúde, ou out of pockets, foi de $6,8 \%$ da renda familiar no decil mais pobre, e 3,1\% no mais rico, apresentando uma distribuição regressiva que, segundo os nossos dados, se manteve na versão 2008/2009 da POF. Já os gastos das famílias brasileiras com saúde, em 2002/2003, eram superiores a $10 \%$ da renda familiar, situação que se manteve segundo os dados de nosso estudo. Além disso, mediante o uso da FIA efetiva, especificamente com o índice de Kakwani, foi possível analisar melhor a distribuição dos pagamentos feitos com impostos diretos que contribuem no financiamento do SUS, os quais se apresentam como progressivos nas curvas de concentração e na FIA estrutural, sendo também avaliados dessa forma em 2002/2003 por Ugá e Santos (19). Porém, por ter um índice de Kakwani próximo à zero, agora sabemos que são proporcionais, ou seja, que toda a população pagou uma proporção similar nessa fonte de financiamento. Embora este não seja o pior cenário, também não é o melhor, ou ideal, para obter uma cobertura de proteção financeira.

Da mesma forma, para a consolidação das três fontes aqui analisadas, o índice de Kakwani contribui com uma análise mais detalhada, fornecendo um índice próximo do zero, 
que apesar de ser negativo e teoricamente poder ser considerado como regressivo, consideramos como proporcionais a consolidação das fontes de financiamento encontradas na POF 2008/2009. Já o índice de Gini, presente na FIA efetiva, confirma de forma numérica que os gastos da população brasileira com saúde são ainda menos equitativos $(0,60)$ do que a própria distribuição geral dos recursos $(0,59)$. Isso tem especial magnitude ao saber que o Brasil é um dos países menos equitativos no mundo (31).

\section{Conclusão}

Enquanto o gasto público em saúde não superar o privado, o orçamento público não for suficiente para suprir todas as necessidades do SUS e as contribuições das fontes de financiamento não forem mais progressivas, o aumento da pobreza e as catástrofes financeiras por falta de cobertura de proteção financeira não diminuirão e muito menos desaparecerão, ao contrário, serão um impulsor de outros problemas sociais dentro da sociedade. Daí a importância de garantir um adequado financiamento do SUS e expandir a oferta e qualidade dos serviços, para que os usuários que financiam o sistema via imposto não tenham que procurar planos de saúde privados nem comprar medicamentos para seus tratamentos de forma direta. Também é fundamental modificar as leis para que as companhias de planos de saúde forneçam os medicamentos necessários para todos seus usuários da mesma forma que o SUS faz. Isso contribuiria grandemente para a diminuição do gasto dos usuários no sistema complementar e aliviaria o peso extra que é repassado para o SUS.

\section{Referências}

1. World Health Organization. La financiación de los sistemas de salud [Internet]. 2010. Disponível em: http://www.who.int/whr/2010/whr10_es.pdf?ua=1

2. Asante AD, Price J, Hayen A, Irava W, Martins J, Guinness L, et al. Assessment of equity in healthcare financing in Fiji and Timor-Leste: a study protocol. BMJ Open [Internet]. British Medical Journal Publishing Group; 2014 Dec 2 [Acesso em 2018 out 25];4(12):e006806. Disponível em: http://www.ncbi.nlm.nih.gov/pubmed/25468509

3. Rodney AM, Hill PS. Achieving equity within universal health coverage: a narrative review of progress and resources for measuring success. Int $\mathrm{J}$ Equity Health [Internet]. 2014;13(1):72. Disponível em: http://equityhealthj.biomedcentral.com/articles/10.1186/s12939-014-0072-8 
4. Asante A, Price J, Hayen A, Jan S, Wiseman V. Equity in Health Care Financing in Lowand Middle-Income Countries: A Systematic Review of Evidence from Studies Using Benefit and Financing Incidence Analyses. Ho Y-S, editor. PLoS One [Internet]. Public Library of Science; 2016 Apr 11 [Acesso em 2018 out 24];11(4):e0152866. Disponível em: https://dx.plos.org/10.1371/journal.pone.0152866

5. Pan American Health Organization - PAHO. Health financing in the Americas [Internet]. 2017 [Acesso em 2018 jun 3]. Disponível em: https://www.paho.org/salud-en-las-americas$2017 / ? \mathrm{p}=178$

6. Pan American Health Organization. OPS OMS - OMS publica una nueva edición del informe sobre el monitoreo de los progresos en enfermedades no transmisibles [Internet]. 2018 [Acesso em 2018 maio 11]. Disponível em:

https://www.paho.org/hq/index.php?option=com_content\&view=article\&id=13677\%3Awholaunches-new-ncds-progress-monitor \&catid=740\%3Apress-releases\&ltemid=1926\&lang=es

7. ISAGS. Sistemas de Salud en Suramérica [Internet]. UNASUR, Instituto Suramericano de Gobierno en Salud. Rio de Janeiro; 2012. 27-29 p. Disponível em: http://www.isagsunasur.org/uploads/biblioteca/2/bb[8]ling[2]anx[9].pdf

8. Gwatkin DR, Ergo A. Universal health coverage: friend or foe of health equity? Lancet (London, England) [Internet]. Elsevier; 2011 Jun 25 [Acesso em 2019 maio 10];377(9784):2160-1. Disponível em: http://www.ncbi.nlm.nih.gov/pubmed/21084113

9. O'Connell T, Rasanathan K, Chopra M. What does universal health coverage mean? Lancet [Internet]. Elsevier; 2014 Jan 18 [Acesso em 2019 maio 10];383(9913):277-9. Disponível em: https://linkinghub.elsevier.com/retrieve/pii/S0140673613609551

10. Luiza VL, Tavares NUL, Oliveira MA, Arrais PSD, Ramos LR, Pizzol T da SD, et al. Catastrophic expenditure on medicines in Brazil. Rev Saude Publica [Internet]. Faculdade de Saúde Pública da Universidade de São Paulo; 2016 [Acesso em 2018 abr 10];50(suppl 2). Disponível em: http://www.scielo.br/scielo.php?script=sci_arttext\&pid=S0034$89102016000300302 \&$ Ing=en\&tlng=en

11. Barros AJD, Bastos JL, Dâmaso AH. Catastrophic spending on health care in Brazil: private health insurance does not seem to be the solution. Cad Saude Publica [Internet]. Escola Nacional de Saúde Pública, Fundação Oswaldo Cruz; 2011 [Acesso em 2018 maio 11];27(suppl 2):s254-62. Disponível em:

http://www.scielo.br/scielo.php?script=sci_arttext\&pid=S0102-

311 X2011001400012\&lng=en\&tIng=en

12. Mengue SS, Bertoldi AD, Boing AC, Tavares NU, Pizzol TD, Oliveira MA, Arrais PS, Ramos LR, Farias MR, Luiza VL, Bernal RT. Pesquisa Nacional sobre Acesso, Utilização e Promoção do Uso Racional de Medicamentos (PNAUM): métodos do inquérito domiciliar. Revista de Saúde Pública. 2016;50:4s. 
13. Boing AC, Bertoldi AD, Posenato LG, Peres KG. The influence of health expenditures on household impoverishment in Brazil. Rev Saude Publica [Internet]. Faculdade de Saúde Pública da Universidade de São Paulo; 2014 Oct [Acesso em 2019 maio 7]; 48(5):797-807. Disponível em: http://www.scielo.br/scielo.php?script=sci_arttext\&pid=S0034$89102014000500797 \&$ lng=en\&tlng=en

14. Boing AC, Bertoldi AD, Peres KG. Desigualdades socioeconômicas nos gastos e comprometimento da renda com medicamentos no Sul do Brasil. Rev Saude Publica [Internet]. Faculdade de Saúde Pública da Universidade de São Paulo; 2011 Oct 5 [Acesso em 2018 abr 25]; 45(5):897-905. Disponível em:

http://www.scielo.br/scielo.php?script=sci_arttext\&pid=S0034-

$89102011000500011 \& \operatorname{lng}=$ pt\&tlng=pt

15. Silveira FG, Ferreira J, Acioly L, Calixtre A, Stivali M, Santos MP. Equidade fiscal no Brasil: impactos distributivos da tributação e do gasto social [Internet].

http://www.ipea.gov.br. Instituto de Pesquisa Econômica Aplicada (Ipea); 2011 May [Acesso em 2018 dez 6]. Disponível em: http://repositorio.ipea.gov.br/handle/11058/5207

16. Ataguba JE, Asante AD, Limwattananon S, Wiseman V. How to do (or not to do) ... a health financing incidence analysis, Health Policy and Planning. [Internet]. April 2018 [Acesso em 2018 out 22]; 33(3): 436-444. Disponível em:

https://doi.org/10.1093/heapol/czx188

17. O’Donnell O, van Doorslaer E, Wagstaff A, Lindelow M. Analyzing Health Equity Using Household Survey Data [Internet]. The World Bank; 2007 [Acesso em 2018 dez 27]. Disponível em: http://elibrary.worldbank.org/doi/book/10.1596/978-0-8213-6933-3

18. Instituto Brasileiro de Geografia e Estatística - IBGE. Pesquisa de Orçamentos Familiares - POF 2008-2009 [Internet]. Pesquisa de Orçamentos Familiares - POF 20082009. 2010 [Acesso em 2018 maio 23]. Disponível em: https://www.ibge.gov.br/estatisticasnovoportal/sociais/rendimento-despesa-e-consumo/9050-pesquisa-de-orcamentosfamiliares. $\mathrm{html}$ ?edicao $=9061 \& \mathrm{t}=0$-que-e

19. Ugá MAD, Santos IS. An Analysis of Equity In Brazilian Health System Financing. Health Aff [Internet]. Project HOPE - The People-to-People Health Foundation Inc.; 2007 Jul 2 [Acesso em 2018 out 25];26(4):1017-28. Disponível em: http://www.healthaffairs.org/doi/10.1377/hlthaff.26.4.1017

20. Xu K, World Health Organization. Dept. of Health System Financing Expenditure and Resource Allocation. Distribution of health payments and catastrophic expenditures Methodology. FER/EIP Discuss Pap ; [Internet]. 2005;(no.2.):11 p. Disponível em: http://whqlibdoc.who.int/hq/2005/EIP_HSF_DP_05.2.pdf

21. Kakwani NC. Measurement of Tax Progressivity: An International Comparison. Econ J [Internet]. WileyRoyal Economic Society; 1977 Mar [Acesso em 2018 dez 27];87(345):71. Disponível em: https://www.jstor.org/stable/2231833?origin=crossref 
22. Boing AC, Bertoldi AD, Barros AJD, Posenato LG, Peres KG. Socioeconomic inequality in catastrophic health expenditure in Brazil. Rev Saude Publica [Internet]. Faculdade de Saúde Pública da Universidade de São Paulo; 2014 Aug [Acesso em 2019 maio 15];48(4):632-41. Disponível em:

http://www.scielo.br/scielo.php?script=sci_arttext\&pid=S0034-

$89102014000400632 \& \operatorname{lng}=e n \&$ tIng=en

23. Boccolini CS, de Souza Junior PRB. Inequities in Healthcare utilization: results of the Brazilian National Health Survey, 2013. Int J Equity Health [Internet]. 2016 Dec 17 [Acesso em 2019 maio 14];15(1):150. Disponível em:

http://equityhealthj.biomedcentral.com/articles/10.1186/s12939-016-0444-3

24. Silveira FG, Osório RG, Piola SF. Os gastos das famílias com saúde. Cien Saude Colet [Internet]. ABRASCO - Associação Brasileira de Saúde Coletiva; 2002 [Acesso em 2019 maio 7];7(4):719-31. Disponível em:

http://www.scielo.br/scielo.php?script=sci_arttext\&pid=S1413-

$81232002000400009 \& \operatorname{lng}=$ pt\&tlng=pt

25. Ocké-Reis CO. SUS: o desafio de ser único [Internet]. [cited 2019 May 15]. 176 p.

Available from: https://portal.fiocruz.br/livro/sus-o-desafio-de-ser-unico

26. Ocké-Reis CO, Nogueira da Gama F. Radiografia do gasto tributário em saúde 20032013 [Internet]. Brasilia; 2016 [Acesso em 2018 dez 10]. Disponível em:

http://www.ipea.gov.br/portal/images/stories/PDFs/nota_tecnica/160530_nt_19_radiografia _gasto_tributario_saude_2003_2013.pdf

27. Barbosa Silva OJ. É o Sistema Único de Saúde-SUS para os pobres? Cadernos lberoAmericanos de Direito Sanitario. 2017 Jun 29;6(2):180. Disponível em:

https://www.cadernos.prodisa.fiocruz.br/index.php/cadernos/article/view/391/464

28. Públio RN, Couto BRGM, Valadão AF, Rezende EM. Perfil das solicitações de medicamentos de alto custo ao Sistema Único de Saúde em Minas Gerais. Rev Adm Pública [Internet]. Fundação Getulio Vargas; 2014 Dec [Acesso em 2018 Dec

7];48(6):1567-85. Disponível em:

http://www.scielo.br/scielo.php?script=sci_arttext\&pid=S0034-

$76122014000600010 \& \operatorname{lng}=p t \&$ tIng=pt

29. Câmara dos Deputados. Repercussões da Emenda Constitucional 95/2016 no processo orçamentário [Internet]. Brasília; 2017 [Acesso em 2018 Dec 10]. Disponível em: http://www2.camara.leg.br/atividade-

30. Vieira FS, Pucci De Sá E Benevides R. Os impactos do novo regime fiscal para o financiamento do Sistema Único de Saúde e para a efetivação do direito à saúde no Brasil [Internet]. 2016 [Acesso em 2018 Dec 10]. Disponível em:

http://repositorio.ipea.gov.br/bitstream/11058/7270/1/NT_n28_Disoc.pdf

31. Victora CG, Barreto ML, do Carmo Leal M, Monteiro CA, Schmidt MI, Paim J, et al. Health conditions and health-policy innovations in Brazil: the way forward. Lancet (London, 
England) [Internet]. Elsevier; 2011 Jun 11 [Acesso em 2018 Jan 13];377(9782):2042-53.

Disponível em: http://www.ncbi.nlm.nih.gov/pubmed/21561659

\section{Colaboradores}

Briones MA contribuiu com a concepção e desenho do artigo; análise e interpretação de dados; redação do artigo; revisão crítica de seu conteúdo; e aprovação da versão final. Sousa FP contribuiu com a concepção e desenho do artigo; revisão crítica de seu conteúdo; e aprovação da versão final.

Como citar este artigo:

Briones MA, Sousa FP. Iniquidades nas contribuições das fontes de financiamento da saúde na Pesquisa de Orçamentos Familiares no Brasil. Cadernos Ibero-Americanos de Direito Sanitário. 2020 abr./jun.; 9(2): 54-73.

http://dx.doi.org/10.17566/ciads.v9i2.546 